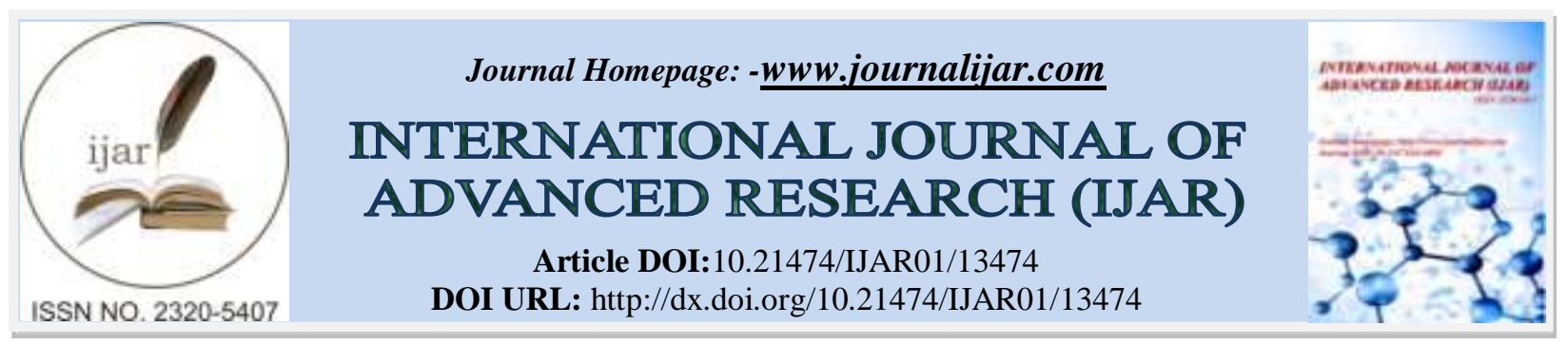

RESEARCH ARTICLE

\title{
HIGH RISK PROSTATE CANCER: COMPARISON OF RADICAL PROSTATECTOMY VERSUS RADIATION THERAPY: EXPERIENCE OF MILITARY HOSPITAL RABAT
}

\author{
Benlemlih M., Hommadi M., Marnouche EA., Maghous A., Bazine A., Andaloussi K., Hadadi K., Sifat H., \\ Elmarjany M. and Mansouri H.
}

Department of Radiotherapy, Mohammed V Military Teaching Hospital, Mohammed V University in Rabat, Morocco.

\section{Manuscript Info}

Manuscript History

Received: 28 July 2021

Final Accepted: 31 August 2021

Published: September 2021

\section{Abstract}

Context: To date, there is no Level 1 evidence comparing the efficacy of radical prostatectomy and radiotherapy for patients with clinicallylocalized prostate cancer.

Objective: to evaluate overall survival (OS) and relapse free survival of high risk prostate cancer (PCa) treated either with external beam radiotherapy (EBRT) associated with androgen deprivation therapy (ADT) or radical prostatectomy (RP) with adjuvant or salvage radiotherapy and ADT. Also, it is very interesting to compare different acute and late toxicity of the both approach.

Materials and Methods: During a period of 10 years from April 2009 and December 2018, 149 patients with high-risk prostate cancer were admitted to the radiotherapy department of the Mohammed V military hospital in Rabat (HMIMV). Among these patients, 17 underwent surgery followed by EBRT and ADT: this is the experimental arm (Group I) and 34 patients who had benefited from treatment with EBRT with ADT were selected from the other patients by a 1: 2 matching method who will represent the reference group (Group II) to allow a comparison with the experimental arm with the minimum possible bias. Results: The overall survival (OS) at 5 and 10 years was $100 \%$ in the both arms while relapse free survival at 5 years was $91,7 \%$ and $93,1 \%$ respectively in group I and II and at 10 years $91,7 \%$ in the group multimodal approach and 88,9\% in the EBRT+ADT group without any significant difference. We note more toxicities in the surgical group with more urinary incontinence $(\mathrm{p}=0,001)$ and more erectile dysfunction.

Conclusion: RP with adjuvant EBRT and EBRT+ADT provided similar long-term cancer control for patients with high-risk prostate cancer but with different toxicity profiles.

Copy Right, IJAR, 2021,. All rights reserved.

\section{Introduction:-}

Following the introduction of prostate cancer (PCa) screening, there has been a rise in the number of men diagnosed with clinically nonmetastatic PCa. Nevertheless, $17-31 \%$ of these men present with high-risk localized or locally advanced disease [1]. The optimal treatment for these patients remains unknown. Historically, men with high-risk

Corresponding Author:- Benlemlih M.

Address:- Department of Radiotherapy, Mohammed V Military Teaching Hospital, Mohammed V University in Rabat, Morocco. 
PCa have been managed most commonly with external beam radiotherapy EBRT, androgen deprivation therapy ADT, or both, while radical prostatectomy RP has been discouraged in this setting, due to concerns about side effects and inadequate disease control. However, no randomized data are available to evaluate RP versus EBRT ADT in terms of survival outcomes and/or toxicity, but the ongoing randomized SPCG-15 trial will provide us with valuable information on this matter [2]. Until these results are available, Patients should at all times be fully informed about all available options, and the likelihood of a multimodal approach including the potential side effects of both local and systemic treatment. The aim of our study is too compare two treatment modalities at high risk prostate cancer concerning oncological outcome and toxicity profile.

\section{Materials and Methods:-}

This is a retrospective study comparing two therapeutic modalities in patients with highriskprostateadenocarcinoma: EBRT associated to ADT versus surgery followed by radiotherapy "multimodal treatment".

During a period of 10 years from April 2009 and December 2018, 149 patients with high-risk prostate cancer were admitted to the radiotherapy department of the Mohammed V military hospital in Rabat (HMIMV). Seventeenpatients(Group I) underwentradical prostatectomy with pelvic lymphadenectomy. EBRT in association with LH-RH analogues was indicated in patients with adverse features (positive margins, T3, post-operative detectable PSA, high grade).EBRT was delivered in 32 - 33 fractions with a total dose of 64 -66 Gray using volumetric modulated arctherapy technique VMAT to prostate fossa. Thirty four patients(Group II) with a ratio 2:1 were selected in the basis of clinical features (similar to group I) and treated with EBRT with long course ADT. EBRT was delivered to pelvis with a total dose of $46-50$ Gy with a boost to prostate of $24-26$ Gy using VMAT.

Statistical analysis of the data was performed by IBM SPSS 26 software. Propensity-score matching was performed. Kaplan-Meier survival analysis was used to compare overall survival and free failure survival. Univariate and Multivariate analysis was used.

\section{Results:-}

\section{Patient's Characteristics}

The frequency of multimodal approach in our series is 11, 4\%. The epidemiological, clinical, paraclinical, and histological characteristics can be summarized in Table 1.

\begin{tabular}{|c|c|c|c|c|}
\hline & & Group I & Group II & $\mathrm{p}$ \\
\hline \multicolumn{2}{|c|}{ AGE (years) } & $65.41 \pm 5,7$ & $68.24 \pm 6,7$ & 0,147 \\
\hline \multicolumn{2}{|c|}{ Initial PSA (ng/ml) } & $10.0[7.3-20.7]$ & $16.1[9.5-23.5]$ & 0,135 \\
\hline \multicolumn{2}{|c|}{ Urinary symptoms $\mathrm{n}(\%)$} & $7(41,2)$ & $17(50)$ & 0,76 \\
\hline \multirow{2}{*}{$\begin{array}{c}\text { Histological } \\
\text { diagnosis } \\
\text { modality }\end{array}$} & Biopsy n (\%) & $16(94,1)$ & $31(91,2)$ & 0,813 \\
\hline & RTUP n (\%) & $10(5,9)$ & $3(8,9)$ & 0,813 \\
\hline
\end{tabular}

Table 2:- The Geason score of the two arms is summarized.

\begin{tabular}{|c|c|c|c|}
\hline Gleason score & Group 1 & Group 2 & P \\
\hline$<7$ & $47.1 \%$ & $20.6 \%$ & 0,242 \\
\hline $3+4$ & $29.4 \%$ & $29.4 \%$ & \\
\hline $4+3$ & $5.9 \%$ & $17.6 \%$ & \\
\hline$>=8$ & $17.6 \%$ & $32.4 \%$ & \\
\hline
\end{tabular}

Table 3:- The TNM classification of our patients in the two groups is shown.

\begin{tabular}{|c|c|c|c|c|}
\hline & Group 1 & \multicolumn{2}{c|}{ Group 2 } & P \\
\cline { 2 - 6 } & $\mathbf{N}$ & $\mathbf{N}$ & $\mathbf{N}$ & 2,9 \\
\hline T2a & 0 & 0 & 4 & 0,66 \\
\hline T2b & 2 & 11,8 & 19 & 55,9 \\
\hline T2c & 13 & 76,5 & 7 & 20,6 \\
\hline T3a & 1 & 5,9 & 3 & 8,8 \\
\hline T3b & 1 & 5,9 & 19 & \\
\hline
\end{tabular}




\begin{tabular}{|c|c|c|c|c|c|}
\hline T4 & 0 & 0 & 0 & 0 & \\
\hline N0 & 17 & 100 & 30 & 88,2 & 0,288 \\
\hline N+ & 0 & 0 & 4 & 11,8 & \\
\hline
\end{tabular}

Surgery in Group I patients consisted of radical prostatectomy associated with lymph node dissection in $82.4 \%$ and radical prostatectomy alone in 17,6 \%. Histopathological data on the operative part showed that the gleason score was greater than that found on the biopsy since an ISUP $4 / 5$ was noted in $23.5 \%$ versus $17.6 \%$, The surgical margins were reached in $70.6 \%$, capsular breach was found in $17.6 \%$, the seminal vesicles were affected in $29.4 \%$ and perineural sheath was noted in $41.2 \%$. As for lymph node dissection, lymph node involvement was found in $14.3 \%$ of patients.

The postoperative PSA median was $0.09 \mathrm{ng} / \mathrm{ml}$ [0.04-0.32].

\section{Oncological Outcomes}

The overall survival (OS) at 5 and 10 years was $100 \%$ in the both arms while relapse free survival at 5 years was $91,7 \%$ and $93,1 \%$ respectively in group I and II and at 10 years $91,7 \%$ in the group multimodal approach and 88,9 $\%$ in the EBRT+ADT group without any significant difference us shown in the survival curves (figure 1)

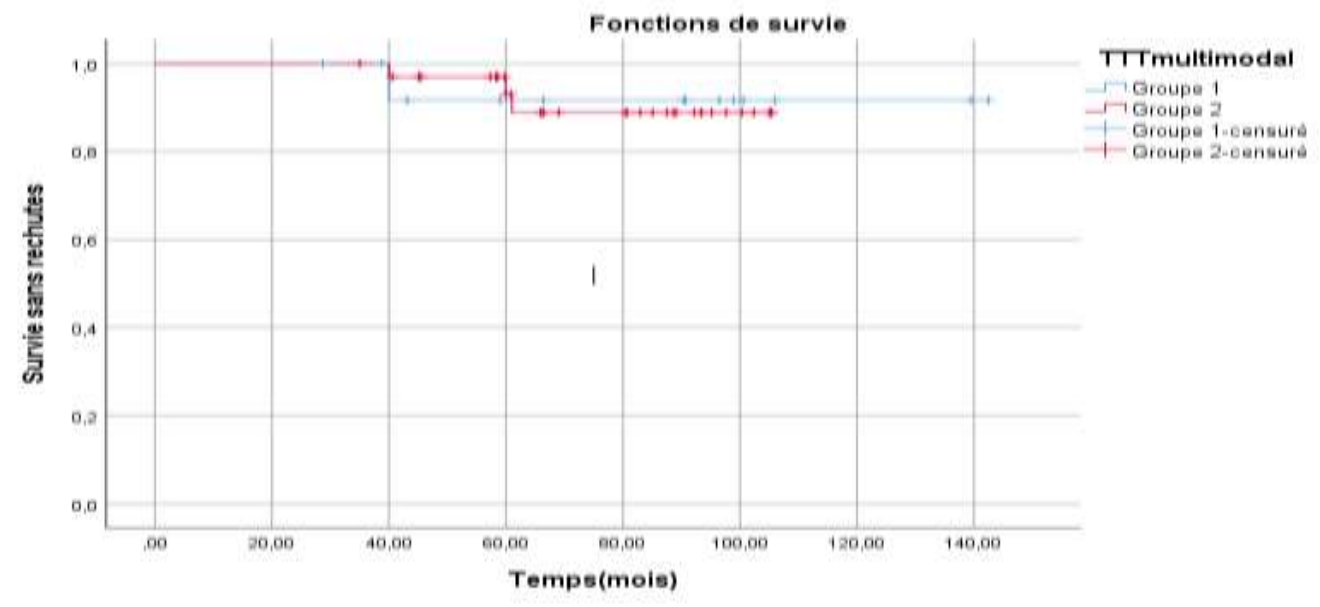

Table 4:- Concerning relapses of our patients, we can summarize the results in the following Table.

\begin{tabular}{|l|l|l|l|l|l|}
\hline Events & \multicolumn{2}{|l|}{ Group I } & Group II & P \\
\hline & $\mathrm{N}$ & $\%$ & $\mathrm{~N}$ & $\%$ & \\
\hline Good locoregional control & 16 & 94,1 & 31 & 91,3 & 0,834 \\
\hline Biochemical relapse & 0 & 0 & 1 & 2,9 & \\
\hline Locoregional relapse & 1 & 5,9 & 1 & 2,9 & \\
\hline Metastatic relapse & 0 & 0 & 1 & 2,9 & \\
\hline
\end{tabular}

\section{Non Oncological Outcomes}

Regarding toxicity after surgical treatment with adjuvant radiotherapy and EBRT-ADT, no statistically significant difference was noted concerning acute toxicity us shown in Table $\mathbf{5}$ while more late complications was noted in the group treated with combining surgery and EBRT-ADT with a $\mathrm{p}=0,001$ for urinary incontinence and a $\mathrm{p}=0,027$ which tends toward a significance concerning erectile dysfunction (Table 6).

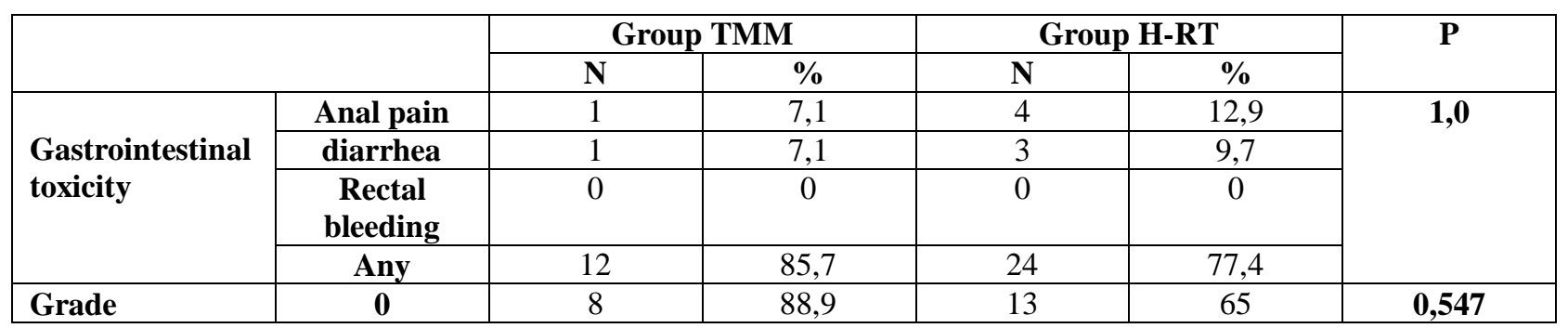




\begin{tabular}{|c|c|c|c|c|c|c|}
\hline & 1 & 0 & 0 & 3 & 15 & \\
\hline & 2 & 1 & 11,1 & 4 & 20 & \\
\hline & 3 & 0 & 0 & 0 & 0 & \\
\hline & 4 & 0 & 0 & 0 & 0 & \\
\hline \multirow{4}{*}{$\begin{array}{l}\text { Genitourinary } \\
\text { toxicity }\end{array}$} & Dysuria & 3 & 21,4 & 3 & 9,4 & \multirow[t]{4}{*}{0,464} \\
\hline & $\begin{array}{l}\text { Mictionnel } \\
\text { Burns }\end{array}$ & 2 & 14,3 & 11 & 34,4 & \\
\hline & Pollakiuria & 7 & 50 & 11 & 43,8 & \\
\hline & Any & 2 & 14,3 & 14 & 12,5 & \\
\hline \multirow[t]{5}{*}{ Grade } & $\mathbf{0}$ & 2 & 16,7 & 4 & 11,5 & \multirow[t]{5}{*}{0,556} \\
\hline & 1 & 1 & 8,3 & 3 & 11,5 & \\
\hline & 2 & 8 & 66,7 & 20 & 76,9 & \\
\hline & 3 & 1 & 8,3 & 0 & 0 & \\
\hline & 4 & 0 & 0 & 0 & 0 & \\
\hline
\end{tabular}

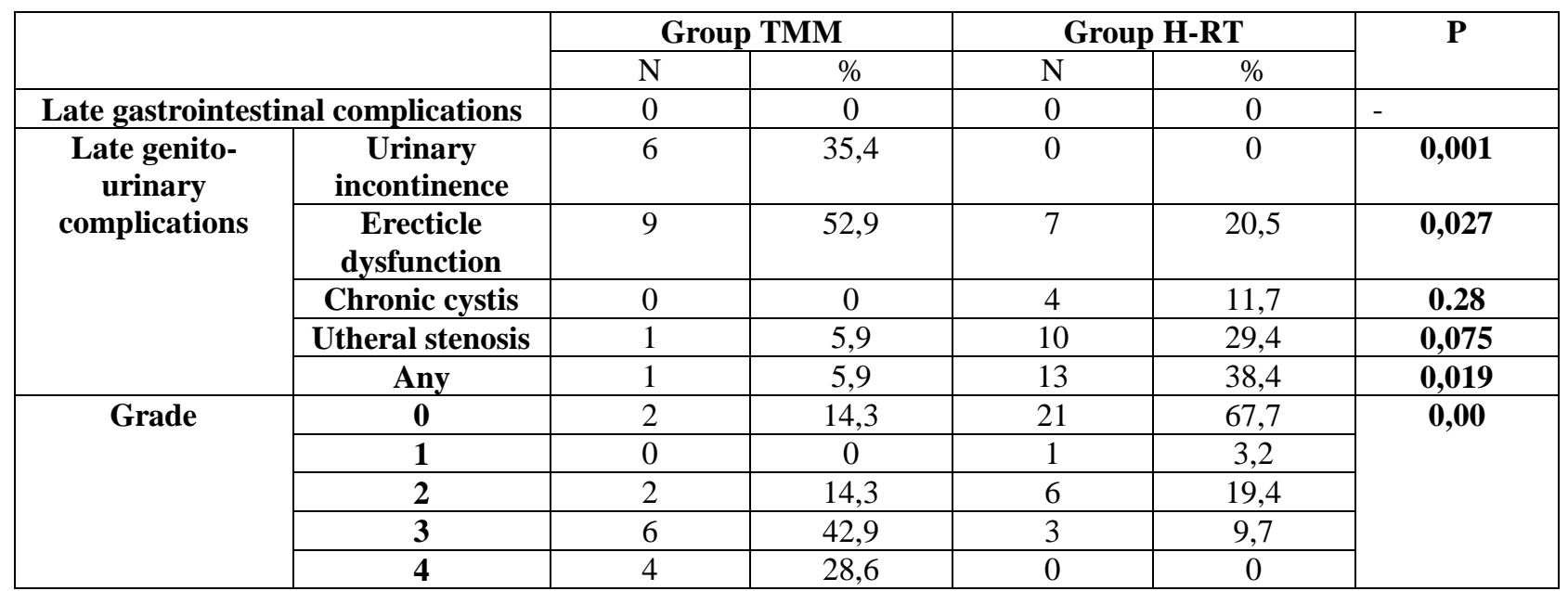

\section{Discussion:-}

Multimodal approach for high risk prostate cancer historically treated with external beam radiotherapy and androgen deprivation therapy is an emergent option and subject of a large debate. It is defined by the association of surgery based on radical prostatectomy and pelvic lymph node dissection followed by adjuvant or salvage radiotherapy with or without ADT [3]. At diagnosis, prostate cancer is usually classified into major risk categories based on TNM clinical stage, biopsy Gleason score, and pretreatment PSA levels. The definition of high risk group is cT2C or ISUP grade $4 / 5$ (Gleason > 7) or PSA $>20 \mathrm{ng} / \mathrm{ml}$ according to the D'Amico classification and the European association of urology (EAU) while the NCCN group define this category by cT3 or ISUP grade $4 / 5$ (Gleason > 7) or PSA > 20 $\mathrm{ng} / \mathrm{ml}$ [4].However, High-risk prostate cancer is an extremely heterogeneous disease and a clear definition of prognostic subgroups based on combinations of unfavorable risk factors is mandatory to dictate the optimal management. So,very studies tried to stratify the high risk patients in prognostic categories like the study of Steven Joniau et al. [5]witch show that survival varied from 49 to $80 \%$ at 5 years according to risk stratification.In fact, there is no large-scale randomized study and the retrospective studies present a lot of bias in particular the heterogeneity of high risk patients, the use of adjuvant treatment, the dose and technique of radiotherapy, the duration of ADT and functional, urinary and sexual status before treatment. It is worth noting that the retrospective series to date that have compared outcomes after surgery and radiation for high-risk tumors have demonstrated widely disparate results, with several groups reporting improved outcomes after radical prostatectomy, others reporting better results after radiation and a few trials noting equivalent efficacy. We start with some institutional comparative studies like this one [6] including 2380 patients with high risk prostate cancer and revealed that the group treated with RP had a lower risk of metastatic progression and a better specific survival. However, an American group of Washington included 6692 high risk prostate cancer [7] and compared overall and specific survival of three arms, RP, brachytherapy and EBRT; the results were in favor of EBRT. Also, a series of Mayo Clinic including 1847 patients [8] find an equivalent specific survival and overall survival at 10 years in the two 
arms like results of our study since there was no statistically significant difference in 5 and 10 years survival as well as free failure survival between the two therapeutic groups. In the literature, there is many metaanalysis with divergent results, like this one [9] published on 2014 including 17 series showing benefice of RP versus EBRT concerning specific and overall survival but an equivalent biochemical free survival between the two groups. Also, a Swedish metaanalysis of observational studies between 1996 and 2010 [10] with an advantage for RP in patients with good general conditions. In fact, several of the meta-analyses of observational studies available demonstrate robust and statistically significant OS and SS benefits in favor of RP over RT-based approaches in all clinically localized disease $[11,12,13,14]$. Indeed, there is a lack of true randomized trial comparing the two treatment modality in a homogeneous high risk population. Through our literature review, in this comparative study [15], long term outcomes appear similar among patients with high risk and very high risk prostate cancer deemed eligible for either RP or EBRT+ADT with similar local recurrence, distant metastasis failure and overall survival.Beyond this, there is equipoise regarding the optimal treatment. Inference must still be drawn from retrospective single-institution studies, population-based studies, and multi-institutional registries, all of which contain inherent selection bias, limited reporting, and potential residual con- founding errors, which are difficult to address even with the best propensity score, instrumental variable, or regression methods and the challenge faced when assembling evidence regarding surgical or radiation-based treatment efficacy for high-risk prostate cancer is the lack of randomized trials to provide sufficient power [16]. Fortunately, SPCG-15 [17] is a prospective, multi-center, open randomized phase III trial. Patients are randomized to either standard (EBRT and ADT) or experimental (RP with extended pelvic lymph-node dissection and with addition of adjuvant or salvage RT and/or ADT if deemed necessary) treatment. Each center follows guidelines regarding the timing and dosing of postoperative RT and adjuvant treatment such as ADT the primary endpoint is cause-specific survival. Secondary endpoints include metastasis-free and overall survival, quality-of-life, functional outcomes and health services requirements. Each subject will be followed up for a minimum of 10 years. Until available results of this interesting prospective study, despite ongoing uncertainly regarding optimal standard of care, NCNN and EAU guidelines list EBRT+ADT, EBRT+brachytherapy or $\mathrm{RP}+\mathrm{PLND}$ combination as valid options for patients with $>5$ years expected survival. Finally, our study evaluated theeffects of local treatment. In general, both radical and systemic treatments result in significant side effects. We can conclude that patients treated with RP experience mainly genitourinary toxicity and sexual dysfunction, while EBRT can result in gastrointestinal problems. Attention should be drawn toincreases the risk of urinary incontinence and erectile dysfunction even no comparison was made between adjuvant and savage radiotherapy or between open and robotic surgery. In our practice, we often underestimate the risk of toxicity and focus on oncological outcomes, however patient can recover from his disease but with a very hard toxicities impacting directly the life quality [18]. Surgery by itself significantly induces a risk of incontinence and deterioration of sexual function [19]. In three main trials of adjuvant radiotherapy, and increased risk of toxicity is consistently noted. In southwest Oncology Group (SWOG) trial [20], the risk of urinary incontinence fell from $2.8 \%$ to $6.5 \%$ urethral stenosis from $9.5 \%$ to $17.8 \%$. Similarly, in the EORTC and ARO trials, the grade 2 or 3 risk is significantly increased [21]. A partial analysis in the EROTC trial did not show difference in postoperative incontinence [22]. In summary, our study is notdevoidof limitations. First, it includes patients treated with RP over a relatively long period of time. Because surgery has been considered as possible a possible first time treatment for selected high risk prostate cancer only in more recent years at our institution, the effective of patients in surgical arm is very low. Also, because most prostate deaths can occur over 10 years after initial treatment, further follow up is needed to provide more robust estimations of prostate cancer specific survival in both arms.

\section{Conclusion:-}

RP with adjuvant EBRT and EBRT+ADT provided similar long-term cancer control for patients with high-risk prostate cancer but with different toxicity profiles. No prospective data is available until now but the the ongoing randomized SPCG-15 trial will provide us with valuable information on this matter.

\section{References:-}

1. [1] Cooperberg MR, Cowan J, Broering JM, Carroll PR. High-risk pros-tate cancer in the United States, 19902007. World J Urol 2008;26:211-8.

2. [2] Stranne J, Brasso K, Brennhovd B, et al. SPCG-15: a prospective randomized study comparing primary radical prostatectomy and primary radiotherapy plus androgen deprivation therapy for lo-cally advanced prostate cancer. Scand J Urol 2018;52:313-20.

3. [3] Hennequin, C et al. (2019). Complications des traitements multimodaux. Progrès En Urologie, 29, S35 S41.

4. [4] NCCN clinical practice guidelines in oncology. Prostate cancer version 3.2020_November 17, 2020 
5. [5] Stratification of High-risk Prostate Cancer into Prognostic Categories: A European Multi-institutional Study Eur Urol 2015 Jan;67(1):157-164. doi: 10.1016/j.eururo.2014.01.020. Epub 2014 Jan 25

6. [6] Metastasis After Radical Prostatectomy or External Beam Radiotherapy for Patients With Clinically Localized Prostate Cancer: A Comparison of Clinical Cohorts Adjusted for Case Mix Michael J. Zelefsky, James A. Eastham, Angel M. Cronin, Zvi Fuks, Zhigang Zhang, Yoshiya Yamada, Andrew Vickers, and Peter T. Scardino J Clin Oncol 2010 Mar 20;28(9):1508-13. doi: 10.1200/JCO.2009.22.2265. Epub 2010 Feb 16.

7. [7] Mortality after prostate cancer treatment with radical prostatectomy, external-beam radiation therapy, or brachytherapy in men without comorbidity. Eur Urol 2013 Sep;64(3):372-8.doi: 10.1016/j.eururo.2013.03.005. Epub 2013 Mar13

8. [8] Long-term survival after radical prostatectomy versus external-beam radiotherapy for patients with high-risk prostate cancer. 2011 Jul 1;117(13):2883-91. doi: 10.1002/cncr.25900. Epub 2011 Jan 10.

9. [9] Radical prostatectomy or radiotherapy in high-risk prostate cancer: a systematic review and metaanalysis. PMID: 24589471 DOI: 10.1016/j.clgc.2014.01.010

10. [10] Comparative effectiveness of radical prostatectomy and radiotherapy in prostate cancer: observational study of mortality outcomes. BMJ. 2014 Feb 26;348:g1502.doi: 10.1136/bmj.g1502.

11. [11] Wallis CJD, Saskin R, Choo R, et al. Surgery versus radiotherapy for clinically-localized prostate cancer: a systematic review and meta-analysis. Eur Urol 2016;70:21-30

12. [12] Roach M, Ceron Lizarraga TL, Lazar AA. Radical prostatectomy versus radiation and androgen deprivation therapy for clinically localized prostate cancer: how good is the evidence? Int J Radiat Oncol Biol Phys 2015;93:1064-70.

13. [13] Petrelli F, Vavassori I, Coinu A, Borgonovo K, Sarti E, Barni S. Radical prostatectomy or radiotherapy in high-risk prostate cancer: a sys-tematic review and metaanalysis. Clin Genitourin Cancer 2014;12:215-24.

14. [14] Serrell EC, Pitts D, Hayn M, Beaule L, Hansen MH, Sammon JD. Review of the comparative effectiveness of radical prostatectomy, radiation therapy, or expectant management of localized prostate cancer in registry data. Urol Oncol 2018;36:183-92.

15. [15] Radical prostatectomy or radiotherapy for high- and very high-risk prostate cancer: a multidisciplinary prostate cancer clinic experience of patients eligible for either treatment. BJU Int. 2019 Nov;124(5):811819.doi: 10.1111/bju.14780. Epub 2019 May 29

16. [16] Comparison of Radical Prostatectomy Versus Radiation and Androgen Deprivation Therapy Strategies as Primary Treatment for High-risk Localized Prostate Cancer: A Systematic Review and Meta-analysis. December 2019 European Urology Focus

17. [17] SPCG-15: a prospective randomized study comparing primary radical prostatectomy and primary radiotherapy plus androgen deprivation therapy for locally advanced prostate cancer. Scandinavian Journal of Urology, DOI: 10.1080/21681805.2018.1520295

18. [18] Sonn GA, Sadetsky N, Presti JC, et al. Differing perceptions

19. of quality of life in patients with prostate cancer and their doctors. J Urol 2013 189; S 59-65; discussion S.

20. [19] Avila M, Patel L, Lopez S, et al. Patient-reported outcomes after treatment for clinically localized prostate cancer: A systematic review and metaanalysis. Cancer treat rev 2018; 66: 23-4

21. [20] Thompson IM, Tangen CM, Paradelo J, et al. Adjuvant radiotherapy for pathological T3N0M0 prostate cancer significantly reduces risk of metastases and improves survival: long-term followup of a randomized clinical trial. J Urol. 2009;181:956-62.

22. [21] Phase III postoperative adjuvant radiotherapy after radical prostatectomy compared with radical prostatectomy alone in $\mathrm{pT} 3$ prostate cancer with postoperative undetectable prostate-specific antigen: ARO 9602/AUO AP 09/95.

23. [22] Van Cangh PJ, Richard F, Lorge F, et al. Adjuvant radiation tehrapy does not cause urinary incontinence after radical prostatectomy: results of a prospective randomized study. J Urol 1998; 159(1): 164-6. 